\title{
Factores de riesgo y diagnóstico citológico del cáncer cervico-uterino
}

\author{
Risk factors and cytological diagnosis of uterine cervical cancer \\ Santes-Bastián María del Carmen ${ }^{1 凶}$, Martínez Díaz Nazaria², Cruz-Ruiz Mireya ${ }^{3}$, Colunga Rodríguez Cecilia ${ }^{4}$, \\ Fernández Sánchez Higinio ${ }^{5}$, Vázquez-Aparicio Mónica ${ }^{6}$ \\ ${ }^{1}$ Facultad de Enfermería. Universidad Veracruzana. Poza Rica. Veracruz. México. Blvd. Lázaro Cárdenas no. \\ 801, col. Morelos, Poza Rica, Veracruz, 93340, tel. 01(782)8245700. \\ ${ }^{2}$ Facultad de Enfermería. Universidad Veracruzana. Poza Rica. Veracruz. México. \\ ${ }^{3}$ Facultad de Psicología. Universidad Veracruzana. Veracruz. Veracruz. México. \\ ${ }^{4}$ Centro Universitario de Ciencias de la Salud. Universidad de Guadalajara. Veracruz, México \\ ${ }^{5}$ Facultad de Enfermería. Universidad Veracruzana. Poza Rica. Veracruz. México. \\ ${ }^{6}$ Facultad de Enfermería. Universidad Veracruzana. Xalapa. Veracruz. México. \\ \Autor para correspondencia: csantes@uv.mx, marycarmen66@hotmail.com
}

Recibido: $13 / 07 / 2018$

Aceptado: 12/09/2018

\section{RESUMEN}

Se analizaron los factores de riesgo y diagnóstico citológico del cáncer cervico-uterino en pacientes de una comunidad de Veracruz bajo un estudio analítico y transversal, en este se incluyó 132 pacientes mayores de 18 años que acudieron al Módulo de Atención a realizarse la citología cervical, las variables fueron factores de riesgo del cáncer cervico-uterino y diagnostico citológico. Se usó como instrumento el "formato de solicitud y reporte de resultados de citología cervical", se utilizó la chi2 para la asociación de los factores de riesgo con el diagnostico citológico. Se halló que el 79.5\% de las mujeres tenían el cuello aparentemente sano, el 9.8\% erosión de cuello, $4.5 \%$ leucorrea, $2.3 \%$ de las mujeres no se observaba el cuello y tenían sangrado anormal, el $.8 \%$ presento cuello anormal y cervicitis Conclusiones: La enfermera debe dar seguimiento a los casos, ya que es el primer contacto del paciente, así pueda brindar educación para la salud a todas las mujeres que acuden a realizarse esta prueba.

Palabras claves: Factores de riesgo, citología, cuello del útero.

\begin{abstract}
We analyzed the risk factors and cytological diagnosis of cervical cancer in patients from a community in Veracruz under an analytical and cross-sectional study, in which 132 patients over 18 years of age who attended the Cervical Cytology Care Module were included. the variables were risk factors for cervical-uterine cancer and cytological diagnosis. The "application format and report of cervical cytology results" was used as instrument, chi2 was used for the association of risk factors with cytological diagnosis. It was found that $79.5 \%$ of women had apparently healthy neck, $9.8 \%$ neck erosion, $4.5 \%$ leucorrhea, $2.3 \%$ of women had no neck and had abnormal bleeding, .8\% had an abnormal neck and cervicitis Conclusions: The nurse must follow up on the cases, since it is the first contact of the patient, so she can provide health education to all the women who come to have this test.
\end{abstract}


Keywords: Risk Factors, cytology, Cervix Uteri.

\section{INTRODUCCIÓN}

El cáncer cervico uterino es un problema de salud pública alrededor del mundo, las tasas más altas de incidencia y mortalidad se presentan en la región de América Latina con 40.28 y el Caribe con 35.78 por cada 100,000 habitantes; siendo solo superado por el este de África y Malasia (Lewis, 2004).

A pesar de contar con métodos de detección oportuna gratuitos para la mayoría de la población en México es un problema de salud que persiste pues según la Dirección General de Información en Salud (DGIS, 2018) 4, 065 mujeres murieron a causa de esta enfermedad a nivel nacional durante el 2016, en el estado de Veracruz se encuentran registradas 340 muertes, siendo el estado número 3 a nivel nacional de mayores descensos, solo después de la ciudad y estado de México.

Actualmente se reconoce que existen factores de riesgo vinculados al cáncer cervico uterino, el principal ligado a este es el virus de infección por Virus del Papiloma Humano (VPH), edad de inicio de las relaciones sexuales, múltiples parejas sexuales, el hábito de fumar, ser mayor de 64 años, haber consumido hormonas anticonceptivas por largos periodos, antecedentes de enfermedades de transmisión sexual entre otros (Centro para el Control y Prevención de enfermedades [CDC], 2018).

La importancia de la detección oportuna a través de la citología cervical radica en la posibilidad de realizar un diagnóstico y atención temprana para las mujeres además de identificar el riesgo de las mujeres a padecer la enfermedad y una ocasión para el personal de salud de implementar estrategias para la modificación de conductas. Debido a la relevancia que representa la prevalencia del cáncer cervico-uterino a nivel estatal, el objetivo del presente estudio es analizar los factores de riesgo y diagnóstico citológico del cáncer cervico-uterino en pacientes de una comunidad de Veracruz.

\section{MATERIAL Y MÉTODOS}

La presente investigación es de corte analítico porque se asociaron los factores de riesgo y el diagnóstico citológico, realizado en una comunidad de Veracruz, durante el periodo 2012- 2015. La población de estudio fue de 132 mujeres que acudieron a la consulta. Los criterios de inclusión fueron pacientes mayores de 18 años y que firmaron el consentimiento informado; se excluyeron pacientes embarazadas; se eliminaron solicitudes incompletas y las muestras reportadas por el patólogo como inadecuadas.

Para obtener los datos de factores de riesgo y diagnostico citológico del cáncer cervico-uterino se utilizó como instrumento el formato de "solicitud y reporte de resultados de citología cervical" emitido por la Secretaria de Salud de México, el cual consta de 4 apartados, que comprenden datos de identificación de la unidad y de la paciente, antecedentes donde se consideran los factores de riesgo, resultados de la citología como la observación y los emitidos por el análisis patológico.

Para la toma de la muestra del Papanicolaou se preparó el material a utilizar como laminillas rotulada con el lápiz diamante, espátula de Ayre, alcohol etílico de $96^{\circ}$, guantes estériles, espejo vaginal desechable, la mesa de exploración, mesa mayo, lámpara de chicote. Para la realización del procedimiento del Papanicolaou primeramente se orientó a la paciente sobre la técnica a realizarse (SSA, 2006) y al término se le informo la fecha probable de entrega de resultados. 
Para la tabulación de los datos se utilizó el programa Excel, el análisis se realizó por medio del programa estadístico SPSS versión 24.0, utilizando la estadística descriptiva, como medidas de tendencia central, porcentajes y frecuencias, así como la estadística inferencial usando la chi2 para la asociación de los factores de riesgo con el diagnóstico citológico del cáncer cervico-uterino.

Para las consideraciones éticas se consultó el artículo 100 y 101 de la Ley General de Salud y el Reglamento de la Ley General de Salud en materia de investigación dado que es una investigación con riesgo mínimo según el artículo 17.

\section{RESULTADOS}

De las 132 pacientes a quienes se les realizó la prueba del Papanicolaou la edad promedio fue de 42.88 años, desviación estándar de 12.97, edad mínima 20 años y máxima de 80 años. La mayoría nació en el estado de Veracruz con un $79.5 \%, 12.9 \%$ en Puebla, el 2.2\% en Hidalgo, el $1.5 \%$ en el Estado de México y en Sinaloa, el $.8 \%$ en Chiapas, Ciudad de México y Oaxaca respectivamente.

De acuerdo a los antecedentes de las pacientes, el $47 \%$ de las mujeres se realiza la citología subsecuentemente, $34.1 \%$ después de 3 años y el $18.9 \%$ es la primera vez que se lo realiza. Los factores de riesgo referidos por las pacientes fueron que el $32.6 \%$ inicio a tener relaciones sexuales antes de los 18 años, 3.8\% presenta antecedentes de Infecciones de Transmisión Sexual (ITS), 3\% tiene múltiples parejas sexuales y el $0.8 \%$ fuma, el $59.8 \%$ de la muestra no presentó ningún factor de riesgo para cáncer cervico-uterino (Tabla. 1).

Tabla 1. Factores de riesgo de las pacientes que se realizaron la prueba del Papanicolaou.

\begin{tabular}{|l|l|l|}
\hline \multicolumn{1}{|c|}{ Factores de riesgo } & \multicolumn{1}{|c|}{ Frecuencia } & \multicolumn{1}{c|}{$\%$} \\
\hline Inicio de relaciones sexuales antes & & \\
de los 18 años & & \\
Múltiples parejas sexuales & 43 & 32.6 \\
Antecedentes de ITS & 4 & 3 \\
Tabaquismo & 5 & 3.8 \\
Ninguno & 1 & 0.8 \\
& 79 & 59.8 \\
\hline Total & 132 & 100 \\
\hline
\end{tabular}

Fuente: Directa

Las características observadas por el personal de enfermería del cuello uterino en las pacientes estudiadas, se halló que el $79.5 \%$ de las mujeres tenían el cuello aparentemente sano, el $9.8 \%$ erosión de cuello, $4.5 \%$ leucorrea, $2.3 \%$ de las mujeres no se observaba el cuello y tenían sangrado anormal, el $.8 \%$ presento cuello anormal y cervicitis (Ver tabla 2). 
Tabla 2. Características observadas del cuello uterino de las pacientes que se realizaron la prueba del Papanicolaou

\begin{tabular}{|l|c|r|}
\hline Características observadas del cuello-uterino & Frecuencia & $\%$ \\
\hline Cuello aparentemente sano & 105 & 79.5 \\
Cuello anormal & 1 & .8 \\
Erosión del cuello & 13 & 9.8 \\
Cervicitis & 1 & .8 \\
Leucorrea & 6 & 4.5 \\
Sangrado anormal & 3 & 2.3 \\
No se observa Cuello & 3 & 2.3 \\
\hline Total & 132 & 100 \\
\hline
\end{tabular}

Fuente: Directa

El $47.7 \%$ de las pacientes que se realizaron la prueba del Papanicolaou presentaron un Diagnostico citológico de cambios reactivos por inflamación, $25.8 \%$ infección por cocobacilos y un $16.7 \%$ atrofia (ver tabla 3 ).

Tabla 3. Diagnostico citológico de las mujeres que se realizaron la prueba del Papanicolaou

\begin{tabular}{|l|c|r|}
\hline \multicolumn{1}{|c|}{ Diagnóstico citológico } & fr & \multicolumn{1}{c|}{$\%$} \\
\hline Dentro del límite normal(negativo a cáncer) & 2 & 1.5 \\
\hline Infección por candidiasis & 1 & .8 \\
\hline Infección por cocobacilos & 34 & 25.8 \\
\hline Infección por actimomyces sp & 3 & 2.3 \\
\hline Cambios reactivos por inflamación & 63 & 47.7 \\
\hline Atrofia & 22 & 16.7 \\
\hline $\begin{array}{l}\text { Lesión escamosa intra-epiterial de bajo grado (LEIBG)/ Displasia leve } \\
\text { /(NIC 1/VPH) }\end{array}$ & 3 & 2.3 \\
\hline Infección por cocobacilos y actimomyces & 1 & .8 \\
\hline Infección por cocobacilos y atrofia & 2 & 1.5 \\
\hline Cambios reactivos por inflamación y atrofia & 1 & .8 \\
\hline Total & 132 & 100.0 \\
\hline
\end{tabular}

Fuente: Directa 
Al analizar los factores de riesgo y diagnostico citológico en las mujeres que se realizaron la prueba del Papanociolao, se encontró una asociación significativa con una $\mathrm{p}=<.015$, principalmente en el factor de riesgo de inicio de relaciones sexuales antes de los 18 años con diagnostico citológico de infección por cocobacilos, cambios reactivos por inflamación y atrofia, seguida de múltiples parejas sexuales y por antecedentes de infecciones de transmisión sexual (ver tabla 4).

Tabla 4. Asociación de los factores de riesgo y diagnostico citológico de las mujeres que se realizaron la prueba del Papanicolaou

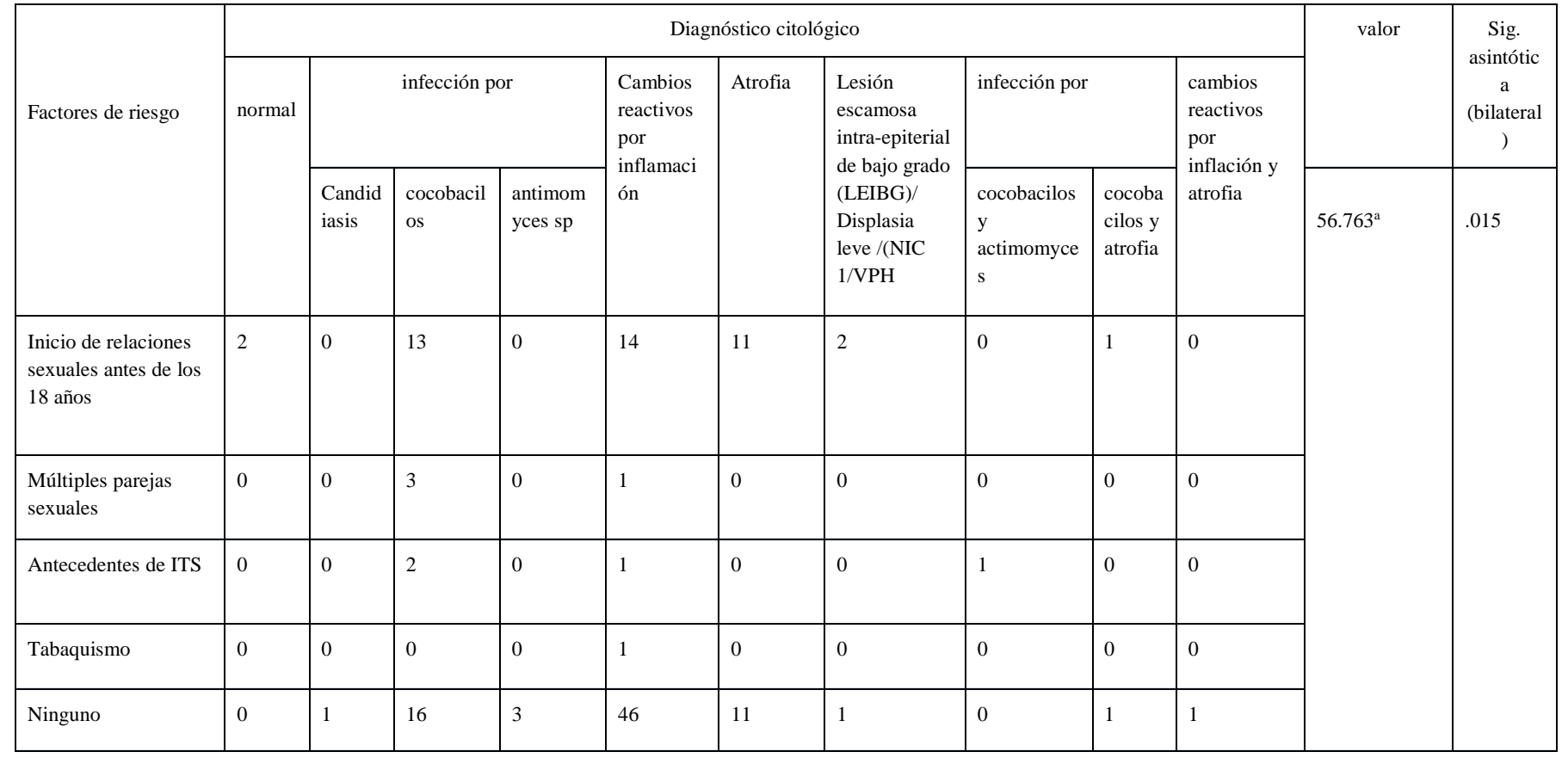

Fuente: Directa

\section{DISCUSIÓN}

Los factores de riesgo referidos por las pacientes fueron; el $32.6 \%$ inicio de relaciones sexuales antes de los 18 años, $3.8 \%$ presenta antecedentes de Infecciones de Transmisión Sexual (ITS), el $3 \%$ tiene múltiples parejas sexuales y el $0.8 \%$ fuma, datos similares a lo reportado por Romero y Rojas (2012) donde refieren que $29 \%$ de las mujeres tenían antecedentes de infección de transmisión sexual, el $1 \%$ no tiene una sola pareja sexual y fumaban de manera ocasional, diferente a lo reportado por Cartaya et al. (2010), que mencionan que el $54.5 \%$ inicio la relaciones sexuales antes de los 16 años, el 58.1\% consumía tabaco, el $74.5 \%$ tiene múltiples compañeros sexuales y el $81.8 \%$ infecciones de transmisión sexual.

La mayoría de la mujeres que se realizaron la prueba del Papanicolaou presenta un Diagnostico citológico de cambios reactivos por inflamación de $47.7 \%$, seguida de infección por cocobacilos de $25.8 \%$, atrofia con un $16.7 \%$ y el $2.3 \%$ lesión escamosa intra-epiterial de bajo grado (LEIBG)/ Displasia leve /(NIC 1/VPH), datos similares a lo reportado Romero y Rojas (2012) que refieren muestras anormales con infección de Transmisión sexual y otros con el $78 \%$, siendo el $34.4 \%$ por cocos bacilos cortos, inflamación inespecífica $.46 \%$. Sin embrago, diferente al estudio realizado por Ruiz et al. (2017), quienes refieren que el $14 \%$ fue positivo 
para lesión escamosa intra-epitelial de bajo grado, así como también en el estudio realizado en un hospital Boliviano donde se reporta casi el $50 \%$ de inflamación en las mujeres estudiadas, seguida de levaduras sugestivas por Candida albicans y una mínima parte cambios sugestivos de displasia (Salas et al., 2013).

Al analizar los factores de riesgo y diagnostico citológico en las mujeres que se realizaron la prueba del Papanicolaou, se encontró una asociación significativa con una $\mathrm{p}=<.015$, principalmente en el factor de riesgo de inicio de relaciones sexuales antes de los 18 años con diagnostico citológico de infección por cocobacilos, cambios reactivos por inflamación y atrofia, seguida de múltiples parejas sexuales y por antecedentes de infecciones de transmisión sexual datos similares a lo reportado por Ruiz et al. (2017), donde reportan asociación entre lesión citológica y $\mathrm{VPH}(\mathrm{p}=0.001)$, así como haber iniciado las relaciones sexuales a los 14 años o menos $(\mathrm{p}=0.024)$.

\section{CONCLUSIONES}

El cáncer cervico-uterino es la segunda causa de muerte en la mujer, en el año 2013 fallecieron 3,771 defunciones con una edad de 25 años y más, siendo las entidades de mayor mortalidad por este padecimiento Morelos (18.6), Chiapas (17.2) y Veracruz (16.4), (SSA, 2015).

Los resultados de esta investigación muestran que uno de los factores de mayor riesgo para padecer cáncer cervico-uterino es tener relaciones sexuales a temprana edad en este caso antes de los 18 años. Una de las características observadas en el cuello uterino en las pacientes se encontró erosión del cuello uterino y leucorrea. El diagnóstico citológico fue en su mayoría por inflamación. Se halló una asociación significativa con los factores de riesgo y el diagnostico citológico principalmente con el factor de riesgo de inicio de relaciones sexuales antes de los 18 años con diagnostico citológico de infección por cocobacilos, cambios reactivos por inflamación y atrofia.

De acuerdo a los resultados obtenidos en esta investigación, es importante que el personal de Salud le dé seguimiento principalmente la enfermera ya que es el primer contacto con el paciente para brindar educación para la salud a todos las mujeres que acuden a realizarse esta prueba, da orientación de acuerdo a los factores de riesgo detectados, así como los resultados de la observación del cuello uterino, siendo uno de datos importante para darle seguimiento al paciente mientras llega el resultado emitido por el patólogo.

\section{LITERATURA CITADA}

Cartaya Olano, Maritza, Hernández Sáez, Isbel, González Sáez, Yoandra, Álvarez Cisneros, Natacha del Pilar. (2010). Factores de riesgo del cáncer cérvicouterino. Revista Archivo Médico de Camagüey, 14(5). Recuperado el 27 agosto 2018 de: http://scielo.sld.cu/scielo.php?script=s ci_arttext\&pid=S1025$02552010000500005 \& \ln \mathrm{g}=\mathrm{es} \& \operatorname{tln} \mathrm{g}=\mathrm{es}$

División de prevención y control del cáncer, Centros para la Prevención y Control de enfermedades. (2018). Factores de cáncer de cuello uterino. Recuperado de:

https://www.cdc.gov/spanish/cancer/ce rvical/basic_info/risk_factors.htm

Dirección General de Información en Salud. (2018). Cubos dinámicos: defunciones por causa [conjunto de datos]. Recuperado de: http://sinba08.salud.gob.mx/cubos/cmo rtalidadxp.htm

Lewis, J. (2004). A situational analysis of cervical cáncer Latin America \& the Caribbean (Washington D.C. Informe 
200337-2895). Recuperado del sitio de internet de Organización Panamericana de la Salud: https://www.paho.org/hq/dmdocument s/2012/SituationAnalysisCCLAC.pdf

Manual de procedimientos para la Toma de la Muestra de Citología Cervical (2006). Centro de Equidad de Género y Salud Reproductiva. Secretaria de Salud [internet]. Recuperado 27 agosto 2018 de:

http://cnegsr.salud.gob.mx/contenidos/ descargas/CaCu/toma_muestra.pdf

Romero Ledezma, Karla Pamela, Rojas Guardia, Javier. (2012). Frecuencia de los factores de riesgo de Cáncer Cervicouterino en mujeres de 14-65 años, Comunidad Ramadas, Provincia Tapacarí-Cochabamba gestión 2012. Revista Científica Ciencia Médica, 15(1), 18-21. Recuperado el 21 agosto 2018

de:

http://www.scielo.org.bo/scielo.php?sc ript=sci_arttext\&pid=S1817-

74332012000100006.
Ruiz-Leud, A. Bazán-Ruiz, S., Mejía, C.R. (2017). Hallazgos citológicos y factores de riesgo en citología cervical anormal en mujeres de pescadores del norte peruano, 2015. Rev Chil Obstet Ginecol, 82(1): 26-34. Recuperado el 27 agosto 2018 de: https://doi.org/10.4067/S0717-7526201 7000100005

Salas Sabates, I., Tejera Ibarra, G., Ricaño Marques, I., Del Prado Fernández, R. (2013). Aspectos epidemiológicos en citologías con resultados anormales en el hospital boliviano "El Torno". Rev. MEDISAN, 17(3):477-83. Recuperado el 27 agosto 2018 de: http://www.redalyc.org/pdf/3684/3684 45013019.pdf.

Secretaria de Salud (8 de septiembre de 2015). Recuperado el 27 agosto 2018 de: https://www.gob.mx/salud/acciones-yprogramas/información-estadistica.

Copyright (c) 2018 Maria del Carmen Santes Bastián, Nazaria Martinez Diaz, Mireya Cruz Ruiz, Cecilia Colunga Rodriguez, Higinio Fernández Sánchez y Mónica Vázquez Aparicio

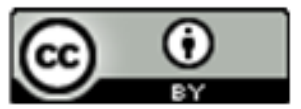

E ste tex to está protegido por una licencia CreativeCommons 4.0.

Usted es libre para Compartir —copiar y redistribuir el $\mathrm{m}$ aterial en cualquier medio o formato- y Adaptar el documento - remezclar, transformar y crear a partir del material- para cualquier propósito, incluso para fines com erciales, siempre que cumpla la condición de:

Atribución: Usted debe dar crédito a la obra original de manera adecuada, proporcionar un enlace a la licencia, e indicar si se han realizado cambios. Puede hacerlo en cualquier forma razonable, pero no de forma tal que sugiera que tiene el apoyo del licenciante o 10 recibe por el uso que hace de la obra.

Resumendelicencia - Textocompletodelalicencia 